\title{
Influence of the Increase in Aluminum Con- centration on the Microstructural Behavior and Hardness of a Cu-Al Alloy (ASTM B-824)
}

\section{Influencia del Aumento de Concentración de Aluminio en el Comportamiento Microestruc- tural y Dureza de una Aleación Cu-Al (ASTM B-824)}

VII International Congress of Science, Technology, Entrepreneurship and Innovation (SECTEI 2020)

Corresponding Author:

L. Castro

lidia.castro@espoch.edu.ec

Published: 26 August 2021

Production and Hosting by Knowledge E

(c) C. Cargua et al. This article is distributed under the terms of the Creative Commons Attribution License, which permits unrestricted use and redistribution provided that the original author and source are credited.

\section{Cargua, M. Moreno, L. Castro, and A. Noguera}

Facultad de Ingeniería Mecánica, Escuela Superior Politécnica de Chimborazo, Riobamba, Ecuador

\section{Abstract}

In this work, the effect of the increase in the concentration of aluminium (Al) on the microstructure and the hardness of bronze to aluminium was studied using optical emission spectrometry, scanning electron microscopy, dispersion spectroscopy techniques of energy, conventional optical microscopy and hardness tests. Alloys with three concentrations by weight of aluminium ( $\mathrm{Cu}-4.5 \mathrm{wt} . \% \mathrm{Al}, \mathrm{Cu}-7 \mathrm{wt} . \% \mathrm{Al}$ and $\mathrm{Cu}-10 \mathrm{wt} . \% \mathrm{Al}$ ) were studied, with the chemical parameters established in the (ASTM B-824), were manufactured and cast in a permanent mold according to the standard (ASTM B-208), to determine the influence of the chemical concentration of aluminium the response surface methodology was used. It was observed that bronzes with a content of $4.5 \mathrm{wt} . \% \mathrm{Al}$ and $7 \mathrm{wt} . \% \mathrm{Al}$, remain with a constant microstructure of phase $\alpha$, and bronzes of $10 \mathrm{wt} . \% \mathrm{Al}$, undergo a change from monophasic to biphasic microstructure $(\alpha+\beta)$ evidencing the appearance of a martensitic microstructure similar to steels providing a better behaviour to resistance to indentation. It is concluded that the increase in the concentration of $\mathrm{Al}$, in the $\mathrm{Cu}-\mathrm{Al}$ alloy, presents a microstructural change, and the appearance of a $\beta$ and $\beta$ 'phase generates a better property in hardness, the hardness has a behaviour $k$ proportional to the aluminium concentration obtaining 53 Brinell value for an increase in the concentration of $5.5 \mathrm{wt} . \% \mathrm{Al}$.

Keywords: alloy, microstructure, bronze, spectrometry, spectroscopy.

\section{Resumen}

En el presente trabajo, se estudió el efecto del aumento de la concentración de aluminio (Al) sobre la microestructura y la dureza del bronce al aluminio, mediante el uso de espectrometría de emisión óptica de chispa, microscopia electrónica de barrido, técnicas de espectroscopia de dispersión de energía, microscopia óptica convencional y pruebas de dureza. En la investigación se estudiaron aleaciones con tres concentraciones en peso de aluminio (Cu-4,5 wt.\% Al, Cu-7 wt.\% Al y Cu-10 wt.\% Al), con los parámetros químicos establecidos en la norma (ASTM B-824), fueron fabricadas y coladas en un molde permanente de acuerdo con la norma (ASTM B-208); para determinar la influencia de la concentración química del aluminio se usó la metodología de superficie de respuesta. Se observó que los bronces con un contenido de 4,5 wt.\% Al y 7 wt.\% Al, permanecen con una microestructura constante de fase $\alpha$, y los bronces de 10 wt.\% Al, sufren un cambio de microestructura monofásica 
a bifásica $(\alpha+\beta)$ evidenciando la aparición de una microestructura martensítica similar a los aceros aportando un mejor comportamiento a la resistencia a indentación. Se concluye que el aumento en la concentración de Al, en la aleación Cu-Al, presenta un cambio microestructural, y la aparición de una fase $\beta$ y $\beta$ ' genera una mejor propiedad en la dureza, la dureza posee un comportamiento k proporcional a la concentración de aluminio obteniendo en valor de 53 Brinell para un incremento en la concentración de 5,5 wt.\% Al.

Palabras Clave: aleación, microestructura, bronce, espectrometría, espectroscopia.

\section{Introducción}

En la actualidad los bronces cuproaluminio han sustituido a ciertas aleaciones de cobre, que están presentes comúnmente en elementos de bombas y válvulas, a la vez presentan una ventaja competitiva a los aceros inoxidables y aleaciones con base níquel. El uso principal de los bronces aluminio de 10 wt.\% Al, está en las construcciones para aplicaciones marinas por su alta resistencia mecánica y excelentes propiedades mecánicas [1].

Los bronces al aluminio son aleaciones en las cuales el aluminio predomina como aleante principal, esta aleación puede contener otros elementos aleantes como son el hierro, níquel, manganeso [2]. En la Figura 1, se evidencia el diagrama de fases de la aleación cobre-aluminio, del lado del cobre se observa que la máxima solubilidad del aluminio en la solución sólida $\alpha$ es aproximadamente del 9,5 wt. $\%$ a $566^{\circ} \mathrm{C}$, por otro lado, a esta temperatura la fase $\beta$ sufre una reacción eutectoide dando origen a la mezcla $(\alpha+\gamma 2)$ [3]. La mayoría de bronces al aluminio comerciales contienen entre 4 y 11 wt.\% de aluminio [4].

El aluminio destaca por ser el elemento que más aumenta la resistencia mecánica del cobre, esto se debe al endurecimiento por solución sólida que se da debido a que ambos metales son de idéntico sistema de cristalización, FCC, y de radios atómicos similares, siendo para el cobre 1,35 A y para el aluminio 1,25 ̊̊ [6].

Aquellas aleaciones de alto contenido de aluminio presentan dificultad a ser mecanizadas por la presencia de alúmina libre. Las aleaciones que contienen menos del $4 \%$ de aluminio se pasivan casi completamente con el cobre, pero aquellas que están por encima del $8 \%$ se las evita debido a que pueden formar porosidades a través riguroso control en la solidificación de la aleación. Las aleaciones que contienen aluminio al 5 y $8 \%$ se incluyen en este campo, aunque solo la primera aleación es fácilmente susceptible de operaciones de conformación en frío. La aleación del 8\% contiene 


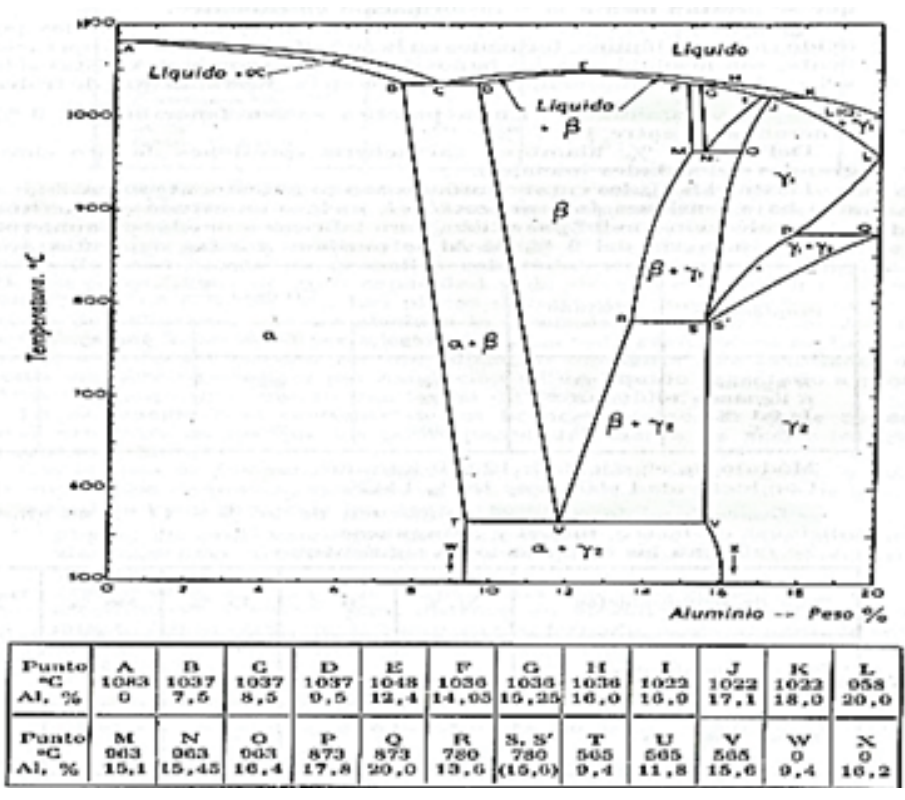

Figure 1

Diagrama de estado Cu-Al del lado del cobre. Fuente: Referencia [5].

hasta un $2 \%$ de hierro, lo que provoca un ligero endurecimiento por precipitación, pero esencialmente es una aleación monofásica [7].

La aleación de aluminio al $8 \%$ se ha utilizado ampliamente para placas de tubos de intercambiador de calor en la industria del petróleo, pero la experiencia naval sugiere un rendimiento de corrosión variable cuando se usa junto con tubos de cobre-níquel 70-30 debido a la corrosión galvánica.

A pesar de investigaciones exhaustivas, la causa subyacente de la resistencia a la corrosión variable no se ha identificado, pero parece estar relacionada con el historial de trabajo en caliente de la aleación y la distribución de la fase inter-metálica FeAl3. El agrietamiento por corrosión por tensión intercristalina se ha experimentado en la aleación del 8\% $\mathrm{Al}$ en atmósferas de vapor sobrecalentado [8], muestran que esto se puede superar con $0,2-0,3 \%$ de adiciones de plata o estaño. Otros trabajos sugieren que el agrietamiento por corrosión bajo tensión en el vapor se ve afectado por pequeñas cantidades de zinc en la aleación.

Turhan y Aksoy [9], han estudiado los efectos de la adición de manganeso en su microestructura y propiedades mecánicas y concluyen que, la adición de manganeso en el material ha aumentado su vida a fatiga al evitar la generación de fases como (Zn, $\mathrm{Cu}) \mathrm{S}$. sin embargo, la resistencia al desgaste se ve disminuida considerablemente al inicio de la prueba de desgaste y recomienda este tipo de aleaciones en elementos en el cual la vida a fatiga juegue un papel crucial.

Ante el interés industrial de disponer de aleaciones con mejores propiedades mecánicas y debido a la gran aplicabilidad de los bronces al aluminio, se estableció 
el objetivo de analizar la influencia de la concentración del aluminio en dichas propiedades, en especial en la dureza. Para ello, es necesario analizar y sintetizar cómo el aumento en la concentración de algunos elementos aleantes como el aluminio mejoran las características físico-mecánicas del material, los resultados de esta investigación podrán ser empleados como base para otros trabajos y de ser factible un aporte a la industria.

En la presente investigación, se presentan los resultados luego de varios meses de trabajo distribuidos en la manufactura, pruebas de rendimiento a dureza y análisis del cambio microestructural, para examinar la influencia del incremento en la concentración de Al, en las propiedades microestructurales y de dureza del bronce al aluminio se usó técnicas de espectrometría de emisión óptica de chispa (OES), microscopia electrónica de barrido (SEM), técnicas de espectroscopia de dispersión de energía (EDS), microscopia óptica convencional y la metodología de superficie de respuesta (RSM) para relacionar los componentes químicos con las pruebas de dureza mediante una ecuación.

\section{Metodología}

Para la influencia en la microestructura se realizarán métodos de pruebas de microscopia óptica convencional y microscopia electrónica de barrido SEM, realizando un análisis cuantitativo y cualitativo, para la dureza se usará un método numérico denominado metodología de superficie de respuesta (RSM), en función a los elementos aleantes y al incremento de aluminio.

Todas las aleaciones de Cu-Al presentan una alta contracción en la solidificación de un $6 \%$ y en el enfriamiento $1,8 \%$, abundante escoria y oxidación del aluminio debido a la diferencia de puntos de fusión que siendo el cobre el metal con un mayor punto de fusión igual a $1083^{\circ} \mathrm{C}$ [10], dando lugar a la formación de óxidos como alúmina, porosidades y rechupes, se consideraron las características químicas que recomienda el estándar ASTM B-824, para las aleaciones de cobre.

\subsection{Materiales}

Los materiales a utilizar son inicialmente cobre y aluminio en condiciones de suministro, la mayoría de bronces al aluminio comerciales poseen los rangos de aluminio entre el (4-11 wt.\%), se preestableció los rangos de contenido de acuerdo a la Tabla 1. Para asegurarnos lo ideal es realizar los cálculos con el porcentaje más alto del elemento aleante ya que el aluminio es más vulnerable a sufrir pérdidas, debido a su punto de fusión bajo respecto al cobre. 


\section{Table 1}

Rangos para las aleaciones a fabricar.

Aleación 1
Elemento
$\mathrm{Cu}$
$\mathrm{Al}$
Otros

\%
Restante
$4-6$
$\geq 2$

Aleación 2
Elemento
$\mathrm{Cu}$
$\mathrm{Al}$
Otros

$\%$
Restante
$7-9$
$\geq 2$

Aleación 3
Elemento
$\mathrm{Cu}$
$\mathrm{Al}$
Otros

$\%$

Restante

$10-12$

$\geq 2$

Para la obtención de los ejes se estiman los efectos de contracción volumétrica de un 4\% en la fundición (ver Tabla 2). De acuerdo, a estos parámetros se estima el contenido de la Tabla 3. La disposición general del material a fundir se describe en la Tabla 4.

\section{Table 2}

Coeficientes de contracción del metal.

Metal o aleación
Fundición Gris
Fundición blanca
Fundición nodular
Aceros No Aleados
Aceros muy aleados
Bronces de Sn
Bronces de Al
Latones
Cobre
Aluminio
Aleaciones ligeras
Aleaciones de Mg
Níquel
Cuproníquel
Cinc

\% de contracción
$3-5$
$6-7$
$4-5$
$5-7$
$8-10$
$5-7$
$4-5,5$
$6-7$
$4-5$
$5-7$
$5-8$
$4-5$
$5-6$
$5-5,5$
$4-4,5$

Fuente: Referencia [11].

\section{Table 3}

Concentración volumétrica y dimensiones totales.

\begin{tabular}{|l|l|l|l|l|} 
& $\begin{array}{l}\text { Dimensiones } \\
(\mathbf{m m})\end{array}$ & iniciales & $\begin{array}{l}\text { Contracción } \\
(\%)\end{array}$ & $\begin{array}{l}\text { volumétrica } \\
\text { Diámetro total }\end{array} 14$ \\
\hline Longitud total & 110 & 4 & $\begin{array}{l}\text { Dimsiones } \\
\text { finales }(\mathbf{m m})\end{array}$ \\
\hline
\end{tabular}


Table 4

Disposiciones generales del material a fusión.

\begin{tabular}{|c|c|c|c|c|}
\hline $\begin{array}{l}\text { Ensayos por } \\
\text { composición }\end{array}$ & Numero de ejes & $\begin{array}{l}\text { Diámetro de eje } \\
(\mathrm{cm})\end{array}$ & Longitud de eje $(\mathrm{cm})$ & $\begin{array}{l}\text { Volumen de los ejes } \\
\text { (cm3) }\end{array}$ \\
\hline $\begin{array}{l}\text { Probetas } \\
\text { resistencia a } \\
\text { indentación y } \\
\text { microscopia }\end{array}$ & 1 & 2 & 16 & 50,265 \\
\hline Total & & & & 50,265 \\
\hline
\end{tabular}

\subsection{Fusión del material}

De acuerdo al volumen a fundir para cada aleación y sus rangos se procede con el cálculo de la masa requerida para cada elemento a diferente concentración, esto se realiza mediante la ecuación [1] pues permite el cálculo de la masa a fundir en función de las densidades de cada material, que son datos conocidos y además del volumen que se requiere de aleación que también es un dato que lo elige el investigador. (donde $m_{\text {!"’!\# }}$ es la masa del elemento a fundir, $\rho_{\text {?"!! }}$ es la densidad del elemento a fundir, \%!"!:\# es el porcentaje del elemento a fundir, $v_{\$ \% \$ \& " ~ \& " ! \& ~}$ es el volumen total de la aleación).

Las densidades de cada material son $8,93 \mathrm{~g} / \mathrm{cm}^{3}$ para el cobre y $2,69 \mathrm{~g} / \mathrm{cm}^{3}$ para el aluminio [12].

$$
m_{\text {elem }}=\rho_{\text {elem }} \times\left(\% \text { elem } \times v_{\text {total alea }}\right) .
$$

A partir de la Ecuación (1), se obtienen los valores en peso de cada elemento para la producción de las tres coladas, los contenidos se presentan en la Tabla 5.

\section{Table 5}

Contenido en masa de Cu y Al para cada colada.

\begin{tabular}{|l|l|l|l}
\hline Colada & $\mathbf{1}$ & $\mathbf{2}$ & $\mathbf{3}$ \\
\hline Masa Cu (g) & 424,30 & 410,76 & 397,22 \\
\hline Masa Al (g) & 8,14 & 12,21 & 16,29
\end{tabular}

La cantidad de calor necesaria para que una sustancia de masa m cambie su estado, calcula partir de la Ecuación (2), sabiendo que el calor latente de fusión de la sustancia es de $214 \mathrm{~kJ} / \mathrm{kg}$, para el cobre. El calor requerido para la fusión del cobre se expresa en la Tabla 5.

$$
Q=m \times L_{F},
$$

donde: Q: Calor ( J); Lf: Calor latente de fusión de la sustancia $(\mathrm{kJ} / \mathrm{kg})$.

Para llevar a la fusión a esta cantidad de masa se utilizará un horno de gas con un quemador (DFC) de capacidad de $10 \mathrm{~kg}$ y una potencia media de $53,74 \mathrm{~kW} / \mathrm{h}$ a una 
presión de salida de 25 PSI. El crisol debe ser necesariamente de arcilla o grafito debido a que la mayoría de hornos son higroscópicos y absorben el agua hasta 5-10\% de su peso., por lo que al utilizar crisoles de grafito para fundir aleaciones no ferrosas estos son bastante permeables al vapor de agua o hidrogeno producido por la combustión, ya que están diseñados con un $40 \%$ porosos [13].

\subsection{Diseño del sistema de alimentación}

El proceso de fundición que se utilizará es por gravedad, en moldes permanentes por lo general se utiliza moldes metálicos también denominados coquillas, generalmente se usan para fundir piezas de bajo punto de fusión como aluminio, zinc y aleaciones de cobre [14]. Este proceso presenta ventajas y desventajas frente al proceso de fundición en arena.

El material apropiado para la construcción moldes permanentes es un acero para trabajos en caliente, como lo recomienda el manual de Ivan Bohman deben ser aleaciones de cromo-molibdeno-vanadio que en Ecuador se denominan AISI H13 [15].

Para la velocidad de vertido se partir a través de la ecuación de continuidad de Bernoulli que establece que la suma de energías en dos puntos cualquiera de un líquido que fluye es igual [16], donde: P: Presión del líquido ( $\mathrm{kPa}$ ); v: Velocidad del flujo [m/s]; g: Gravedad [m/s²]; f: Perdidas por fricción (k).

$$
\frac{P_{1}}{\gamma}+\frac{V_{1}^{2}}{2 g}+h_{1}+f=\frac{P_{2}}{\gamma}+\frac{v_{2}^{2}}{2 g}+h_{2} .
$$

De la Ecuación (3), se obtiene la Ecuación (4), donde: v: Velocidad de vertido (m/s); g: Gravedad $\left(\mathrm{m} / \mathrm{s}^{2}\right)$; h altura $(\mathrm{m})$; entre el pico de descarga de la cuchara de vertido de colada al vertedero [17].

$$
v=+\overline{2 g h} .
$$

Para el tiempo de llenado se usó la ecuación de caudal [5], donde: $\vec{v}$ : Velocidad (m/s); A: Área del canal de alimentación $\left(\mathrm{m}^{2}\right)$.

$$
\begin{gathered}
Q=\overrightarrow{v A}, \\
\text { Tllen }=\frac{V p}{Q} \times k .
\end{gathered}
$$

Se considera que el factor $\mathrm{k}=2$, para compensar la variación del chorro de metal fundido al ingresar al molde. Para determinar el tiempo de solidificación se utiliza el concepto de la regla de Chvorinov's [18]

$$
t_{s}=B\left(\frac{V}{A}\right)^{2},
$$


donde: ts: Tiempo de solidificación(s); V: Volumen de la pieza colada $\left(\mathrm{m}^{3}\right)$; A: Área de la superficie de la pieza colada en contacto con el molde $\left(\mathrm{m}^{2}\right)$; B constante del molde $\left[\mathrm{min} / \mathrm{cm}^{2}\right]$

$$
B=\left[\frac{\rho m}{(\Delta T)}\right]^{2}\left[\frac{1.854 L_{f}}{4 \times k \times \rho \times c}\right]\left[1+\left(\frac{c_{m} \Delta T_{s}}{d^{2}}\right)\right],
$$

donde: $\square m$ : Densidad del metal $\left(\mathrm{kg} / \mathrm{m}^{3}\right)$; Lf: Calor latente de fusión de la sustancia $(\mathrm{kJ} / \mathrm{kg})$; $\Delta \mathrm{T}$ : (Tm-To) ( $\left.{ }^{\circ} \mathrm{K}\right)$; siendo Tm: Temperatura de fusión/solidificación ( $\left.{ }^{\circ} \mathrm{K}\right) ;$ To: Temperatura inicial del molde ( $\left.{ }^{\circ} \mathrm{K}\right)$; $\mathrm{k}$ : Conductividad térmica del molde $\left(\mathrm{w} / \mathrm{m}^{\circ} \mathrm{K}\right)$; $\rho$ : Densidad del material del molde $\left(\mathrm{kg} / \mathrm{m}^{3}\right)$; c: Calor especifico del molde $\left(\mathrm{J} / \mathrm{kg}^{\circ} \mathrm{K}\right) ; \mathrm{Cm}$ : Calor especifico del material $\left(\mathrm{J} / \mathrm{kg}^{\circ} \mathrm{K}\right) ; \Delta \mathrm{Ts}$ : Variación de temperatura del material y temperatura de vertido ( $\left.{ }^{\circ} \mathrm{K}\right)$.

Según Rodríguez C [19] define algunos parámetros de solidificación de una aleación bronce al aluminio, dando como resultado que las aleaciones bronce aluminio pasan de estructuras columnares a equiaxiales alcanzando gradientes de temperatura mínimos e incluso negativos, lo que sugiere tomar en cuenta el momento de la fusión del material ya que dependiendo de la forma del grano la aleación tendrá su aplicabilidad diferente.

\subsubsection{Modelado CAD}

El modelado CAD trata de digitalizar la información física de la pieza en estudio a base de disposición generales establecidas en la Tabla 3. Se usó el software SolidWorks ${ }^{\circledR}$ como herramienta un procesador Intel Core i7 con 16 Gb de memoria RAM.

\subsection{Caracterización y pruebas de rendimiento a dureza}

Los especímenes fueron preparados para caracterización metalográfica a un diámetro de 1,5 cm; Espesor = 0,5 cm, la preparación de la superficie de la muestra se hizo con el estándar ASTM E-3, Para la técnica de experimentación denominada microscopia es necesario revelar la microestructura del material con el fin de analizar y diferenciar las fases presentes en cada concentración de aluminio de cada aleación, el grabado fue realizado con la solución número 40 y 35 del estándar ASTM E-407 (la gravimetría de cada reactivo viene expresada en el estándar mencionado). Las composiciones se muestran en la Tabla 6.

El uso de espectrometría de emisión óptica de chispa se usó para determinar las composiciones químicas de cada colada fabricada, las técnicas SEM Y EDS se utilizaron para el análisis metalográfico. El rendimiento de la dureza de la superficie se realiza imprimiendo en la muestra con un marcador o indentador de determinada geometría, bajo una carga estática conocida que se aplique directamente o por medio de un sistema de palanca determino por el método Brinell que consiste en el uso de una 

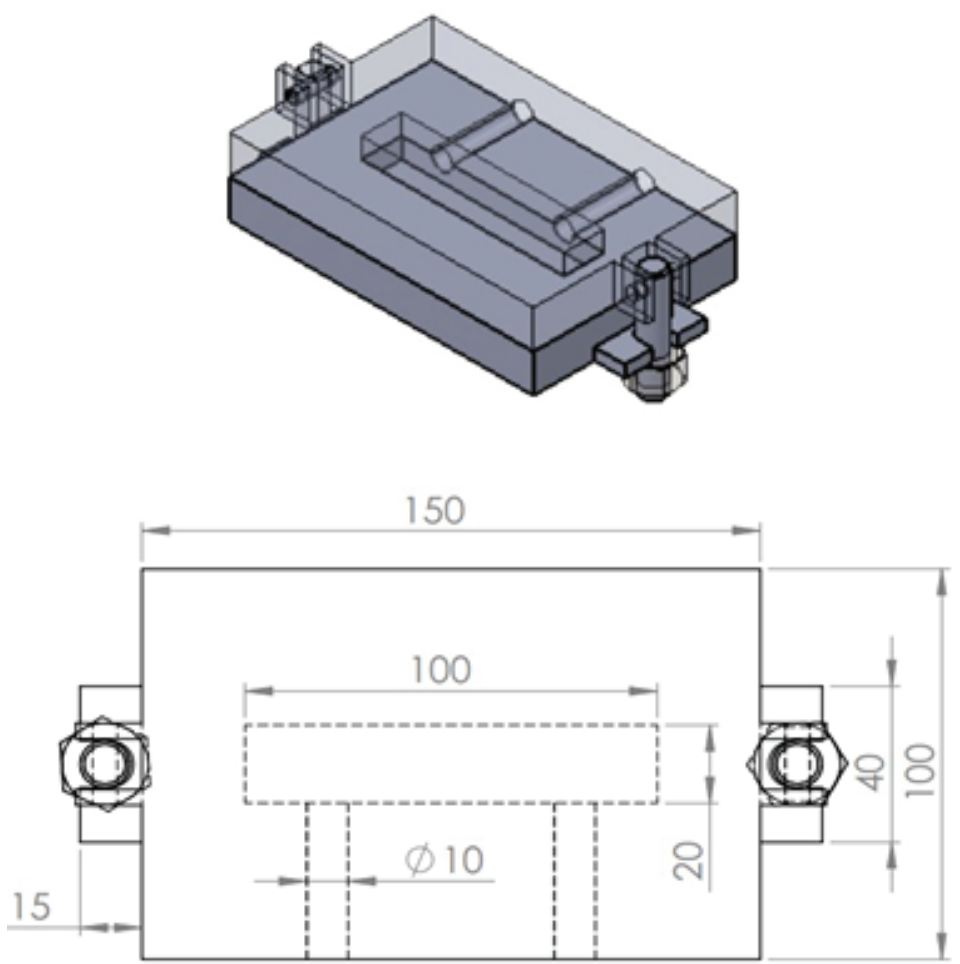

Figure 2

Modelado CAD del molde permanente.

Table 6

Composición de los grabadores 40 y 35 .

\begin{tabular}{|c|c|c|c|}
\hline Número & Reactivo & Cantidad & Procedimiento \\
\hline \multirow[t]{3}{*}{40} & $\mathrm{FeCl} 3$ & $5 \mathrm{~g}$ & $\begin{array}{l}\text { Sumerja o frote unos } \\
\text { pocos segundos a la vez. }\end{array}$ \\
\hline & $\mathrm{HCl}$ & $16 \mathrm{ml}$ & \\
\hline & Etanol al 95\% & $60 \mathrm{ml}$ & \\
\hline \multirow[t]{4}{*}{35} & $\mathrm{FeCl} 3$ & $20 \mathrm{~g}$ & $\begin{array}{l}\text { Utilice mascarilla sumerja } \\
\text { o frote unos pocos } \\
\text { segundos a la vez hasta } \\
\text { obtener los resultados } \\
\text { deseados. }\end{array}$ \\
\hline & $\mathrm{HCl}$ & $5 \mathrm{ml}$ & \\
\hline & $\mathrm{CrO} 3$ & $1 \mathrm{~g}$ & \\
\hline & Agua destilada & $100 \mathrm{ml}$ & \\
\hline
\end{tabular}

máquina calibrada, se fuerza una bola fabricada de un acero extraduro, o bien, de carburo de tungsteno, de un diámetro ' $D$ ', bajo condiciones específicas, aplicándole una fuerza ' $P$ ' contra la superficie del material a ensayar durante un tiempo ' $t$ ' dado, apareciendo entonces una huella con forma de casquete esférico de diámetro 'd' sobre el material ensayado [3]. 
El valor medido en el ensayo es este diámetro 'd' del casquete en la superficie del material. Los parámetros del ASTM E-10, aplicando una carga de 62,5 kgf; tiempo de aplicación de $30 \mathrm{sec}$; con una distancia entre cada aplicación de 5 veces la diagonal mayor. Con ayuda de la ecuación [8].

$$
H B=\frac{L}{\left(\frac{\pi D}{2}\right)\left(D-\sqrt{D^{2}-d^{2}}\right)},
$$

donde: L: Carga de prueba (kgf); D: Diámetro de la bola (mm); d: Diámetro de la impresión $(\mathrm{mm})$. El número de dureza Brinell (HB) es la razón de la carga en kilogramos al área en milímetros cuadrados de la impresión En esta prueba se utiliza instrumentos de lectura directa basados en el principio de medición de profundidad diferencial.

\subsubsection{Superficie de respuesta}

Suponiendo que cada valor de Yi buscado se pueda aproximar con alta precisión en función a un diseño de superficies (RSM), definiendo para un modelo cuadrado completo:

$$
\begin{gathered}
Y_{i}=a_{01}+a_{1 i} x_{1}+a_{2 i} x_{2}+a_{3 i} x_{3}+a_{4 i} x_{4}+a_{5 i} x_{5}+a_{6 i} x_{1} x_{2} \\
+a_{7 i} x_{1} x_{3} \\
+a_{7 i} x_{1} x_{4}+a_{8 i} x_{1} x_{5}+a_{9 i} x_{1}^{2}+a_{10 i} x_{2}^{2}+a_{11 i} x_{2} x_{3}+a_{11 i} x_{2} x_{3} \\
+a_{12 i} x_{2} x_{4} \\
+a_{13 i} x_{2} x_{5}+a_{14 i} x_{3}^{2}+a_{15 i} x_{3} x_{4}+a_{16 i} x_{3} x_{5}+a_{17 i} x_{4}^{2}+a_{18 i} x_{4} x_{5} \\
+a_{19 i} x_{5}^{2}
\end{gathered}
$$

La Ecuación (10), expresa a un multinomio con interacción cuadrática, la metodología de respuesta de superficie ha sido ampliamente adoptada en textos sobre quimiometría. RSM consiste en un grupo de técnicas estadísticas y matemáticas que se basan en un ajuste de modelos empíricos a los datos experimentales obtenidos en un diseño experimental.

Con este objetivo, se emplean funciones polinómicas lineales o cuadradas para describir una aproximación del sistema estudiado y, en consecuencia, explorar (modelar y desplazar) las condiciones experimentales hasta su optimización [20].

El principal propósito de aplicar la metodología RSM al proyecto es encontrar al modelo matemático y estadístico que mejor se ajuste para optimizarlo a través de un algoritmo genético continuo GA.

\section{Resultados y Discusión}




\subsection{Resultado del diseño y manufactura}

Para nuestro caso según Askeland [11], recomienda utilizar como mazarota el canal de vertido de acuerdo a esto la altura y el diámetro de nuestra es:

$$
\begin{gathered}
D^{3}=\frac{2\left(V_{P} \times k \times c\right)}{\pi} \\
H=2 D
\end{gathered}
$$

Remplazando con los datos presentes en la Tabla 3, y con un coeficiente de contracción $4 \%$ se tiene, un diámetro interior igual a: D: 6,53 mm; diámetro Ext. 2,5 D: 16,32 mm; H: 32,65 mm.

El caudal y la velocidad de vertido se calculan con las ecuaciones $(4,5)$ :

$$
v=0,44 \frac{m}{s}<1 \frac{m}{s}
$$

Según Campbell [13], la velocidad de vertido adecuada para que el metal fundido fluya sin generar defectos por turbulencia debe estar dentro de los rangos de 0,25$0,5 \mathrm{~m} / \mathrm{s}$, si sobrepasa el valor de $1 \mathrm{~m} / \mathrm{s}$, lo mas probable es que las piezas salgan defectuosas, por otro lado Esparza [21] concluye que al reducir la velocidad permite que el sistema de óxidos y otras inclusiones no floten y se mantengan alejadas del frente del metal.

$$
Q=1.16 \times 10^{-5} \frac{m^{3}}{s} .
$$

Remplazando en la ecuación (6) se tiene:

$$
T_{l l e n}=1.4 s \times 2=2,8 s .
$$

Para el cálculo de la constante del molde se debe tener en cuenta que el molde se debe precalentarse a una temperatura de $100-200^{\circ} \mathrm{C}$, con el fin de evitar la solidificación prematura y un flujo del metal adecuado. Por lo tanto, el valor es:

$$
B=45 \frac{s}{\mathrm{~cm}^{2}}
$$

Obteniendo un valor de tiempo de solidificación Ts: 3 sec.

Con los parámetros de las dimensiones obtenidas anteriormente se procede a modelar el macho y las cavidades del molde, el molde se diseñó para que sea desmontable para las el macho y la hembra se sujetan a través de dos pernos hexagonales M 10×35 mm como se indica en la Figura 3.

Bajo parámetros de diseño se modificaron las áreas a un diámetro hidráulico equivalente para una sección cuadrada. 


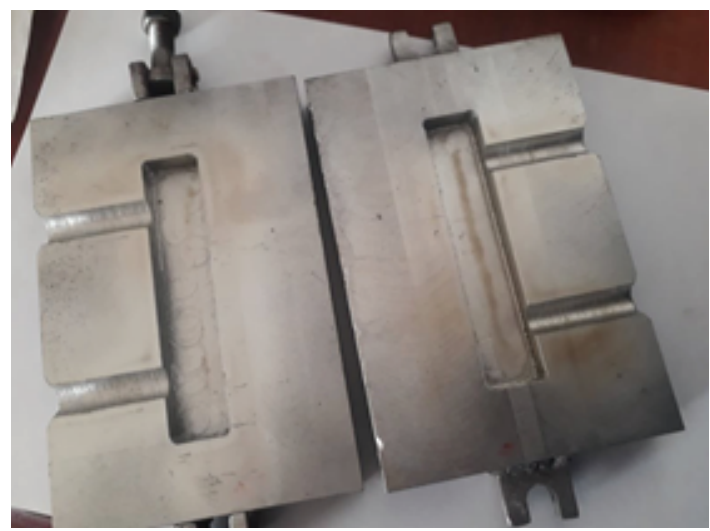

Figure 3

Molde permanente de acero AISI H13.

Acotaciones generales de la manufactura de una aleación Cu-Al:

Debido a que el punto de fusión del aluminio es menor, el aluminio se fundirá mucho antes que el cobre, y para esto es necesario agregar al metal fundido un excedente de aluminio de $8 \%$ a $10 \%$ de la masa ya precalentado para evitar choques térmicos (explosión). Por lo tanto, se deben realizar los siguientes pasos:

- Primero: Cargar dos tercios de la masa total del cobre en el crisol.

- Segundo: Agitar el baño para verificar que todo el cobre este fundido.

- Tercero: Agregar el aluminio en la colada, en este paso hay que tener en cuenta que este elevara la temperatura de la colada por efecto exotérmico, por producto del rápido cambio de estado, por lo que se recomienda precalentarlo antes de agregar directamente a la colada.

- Cuarto: Agregar un tercio restante de cobre sin que haya pasado mucho tiempo después de haber agregado el aluminio, si la colada presenta escoria se recomienda colocar $4 \mathrm{~g}$ de bórax y retirarla con la espátula.

El molde de acero previamente precalentado a $100-200^{\circ} \mathrm{C}$, para evitar el rebote y solidificación prematura en el embudo. Para evitar que en el molde exista un choque térmico y también evitar que la colada se pegue a las paredes del molde es necesario antes de realizar el precalentado del molde aplicar un recubrimiento con pintura de resistencia térmica por lo general esta pintura de resistencia térmica es fabricada con un base de aluminio.

Este recubrimiento ayudara:

(i) Proteger el molde del choque térmico;

(ii) Evitar el enfriamiento prematuro del metal que podría provocar defectos de regazo frio; 
(iii) Confieren cierta permeabilidad superficial a la superficie impermeable para permitir que la masa fundida fluya mejor en la superficie y permitiendo que escape de cualquier condensado.

\subsection{Composiciones de las aleaciones $\mathrm{Cu}-\mathrm{Al}$}

Las composiciones químicas finales obtenidas se muestran en la Tabla 7. Para fines prácticos las aleaciones fueron nombradas como aleación 1,2 y 3. La norma realiza una aclaración que de ser el caso que requiera el fabricante el contenido de aluminio puedo variar acorde a las necesidades que el disponga siempre teniendo en cuenta los puntos de máxima solubilidad del aluminio en el cobre. El espectrómetro que se uso es un Bruker Q2 ION y se observa en la Figura 4.

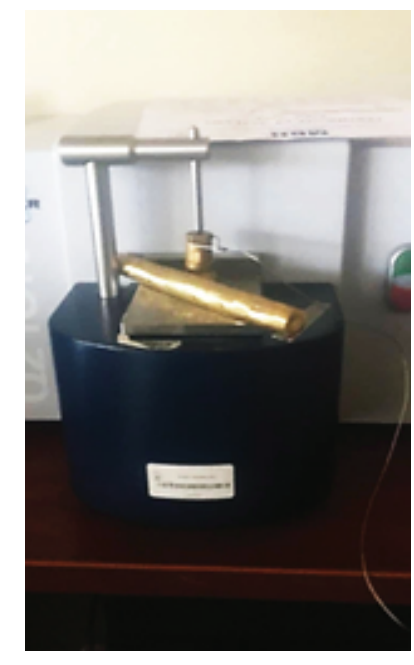

Figure 4

Espectrometría de emisión óptica de chispa OES (Muestra mecanizada).

Table 7

Composiciones químicas finales.

\begin{tabular}{|c|c|c|c|c|c|c|c|}
\hline \multicolumn{8}{|c|}{ Elementos (wt.\%) } \\
\hline $\begin{array}{l}\text { Aleación } \\
\text { No. }\end{array}$ & $\begin{array}{l}\text { Nombre de } \\
\text { la muestra }\end{array}$ & Al & Sn & $\mathrm{Pb}$ & $\mathrm{Fe}$ & $\mathrm{Zn}$ & Mn \\
\hline Aleación 1 & M-1 & 4,536 & 0,135 & 0,094 & 0,082 & - & $<0,003$ \\
\hline Aleación 2 & M-2 & 7,170 & 0,247 & 0,102 & 0,099 & 0,072 & $<0,003$ \\
\hline Aleación 3 & M-3 & 10,110 & 0,232 & 0,119 & 0,075 & 0,648 & $<0,003$ \\
\hline
\end{tabular}

Las composiciones químicas registradas se encuentran bajo los requerimientos generales en composición química que sugiere la ASTM B- 824 para las aleaciones de cobre. 
Table 8

Composiciones químicas generales para las aleaciones de cobre.

Element
Aluminum (Al)
Antimony (Sb)
Arsenic (As)
Carbon (C)
Copper (Cu)
Iron (Fe)
Lead (Pb)
Manganese (Mn)
Nickel (Ni) (incl Colbalt (Co))
Phosphorus (P)
Silicon (Si)
Sulfur (S)
Tin (Sn) O
Zinc (Zn)

\begin{tabular}{l} 
Range or \% max \\
$0.005-13.5$ \\
$0.05-0.70$ \\
$0.0-0.50$ \\
$0.0-0.50$ \\
50.0-99.75 \\
$0.003-1.25$ \\
$0.0-5.0$ \\
$0.002-15.0$ \\
$2.0-30.0$ \\
$0.10-12.0$ \\
$12.0-23.0$ \\
$0.0-5.0$ \\
$0.01-1.0$ \\
$0.005-5.50$ \\
$0.05-0.08$ \\
$0.01-1.0$ \\
$0.50-20.0$ \\
$0.02-2.0$ \\
\hline $2.0-40.0$ \\
\hline
\end{tabular}

Fuente: ASTM B-824.

\subsection{Resultados de la prueba de rendimiento a dureza (Brinell)}

Para las pruebas se usó un durómetro Huayin ${ }^{\circledR}$ HB-3000B con un rengo de fuerza de $187,5-1000$ kgf.

Los resultados de la prueba de rendimiento de la dureza de la superficie de las muestras se dan en la Tabla 9.

Las durezas se calculan a partir de la Ecuación (8), la norma establece que para aleaciones no ferrosas con un espesor e $\leq 5 \mathrm{~mm}$, la carga de debe ser $62,5 \mathrm{kgf}$; diámetro del indentador $\mathrm{d}=5 \mathrm{~mm}$, con un tiempo de aplicación de $30 \mathrm{seg}$, las durezas reportadas en la Tabla 9, son durezas en estado As- Cast, sin tratamiento térmico previo y enfriado en el horno, hay que notar que las durezas no sobrepasan los 100 HBW5/62,5 debido a que no posee elementos aleantes como el $\mathrm{Ni}$ o el Fe en cantidades considerables que refinan el grano mejoran las propiedades mecánicas [22], a esto se puede añadir que la presencia de estaño incrementara considerablemente su resistencia a corrosión [23]. 


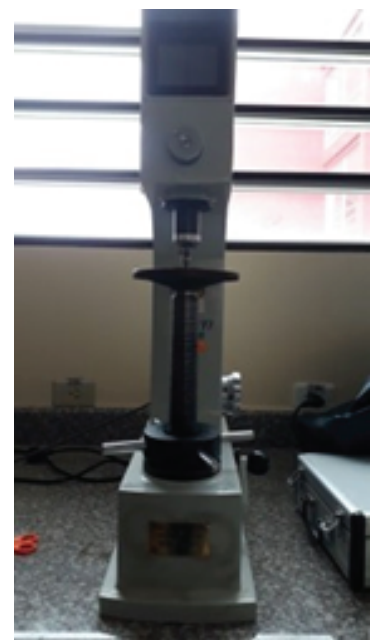

\section{Figure 5}

Durómetro Huayin ${ }^{\circledR}$.

\section{Table 9}

Resultados del ensayo de dureza Brinell.

\begin{tabular}{|c|c|c|c|c|c|c|}
\hline \multirow{6}{*}{$\begin{array}{l}\text { Estado } \\
\text { de la } \\
\text { muestra }\end{array}$} & \multicolumn{2}{|c|}{ Aleación 1} & \multicolumn{2}{|c|}{ Aleación 2} & \multicolumn{2}{|c|}{ Aleación 3} \\
\hline & Al & 4,536 & $\mathrm{Al}$ & 7,170 & $\mathrm{Al}$ & 10,110 \\
\hline & Sn & 0,132 & Sn & 0,247 & Sn & 0,232 \\
\hline & $\mathrm{Cu}$ & Restante & $\mathrm{Cu}$ & Restante & $\mathrm{Cu}$ & Restante \\
\hline & (HBW5/62,5) & PROMEDIO & (HBW5/62,5) & PROMEDIO & (HBW5/62,5) & PROMEDIO \\
\hline & 37,1666 & & 36,0123 & & 84,2102 & \\
\hline (As-Cast) & 31,8833 & 35 & 50,7378 & 43 & 93,7292 & 88 \\
\hline
\end{tabular}

Estos resultados evidencian que a medida que la concentración de aluminio aumenta, el aumento en la dureza es favorecido. Según Rosario S [24], en su investigación concluye que el aumento del contenido con 9,5\% son de menor dureza que las aleaciones con un contenido de 11 wt.\%. Al, confirmando nuestros análisis de resistencia a dureza superficial. 


\subsection{Análisis matemático de los resultados de las pruebas de dureza}

Una vez acabadas las pruebas se determina la regresión multidimensional que permita evaluar los efectos de la composición química de cada aleación, en la propiedad anticorrosiva analizada.

$$
Y,=b_{,-}+b_{,-} f i_{-} X_{(}+\ldots+b_{, l} f_{,} X_{(}
$$

De acuerdo a los datos de dureza a distinto nivel de wt.\% Al recopilados, y a las variables que interviene se realizó un diagrama de dispersión, para ubicar los puntos. La figura muestra la grafica de superficie de los valores de dureza en función a los elementos de aleación descritos en la Tabla 10.

\section{Table 10}

Matriz Principal para el diseño de superficie de respuesta.

\begin{tabular}{|c|c|c|c|c|c|}
\hline \\
\hline & & \\
\hline 4,536 & 0,135 & 0,094 & 0,082 & 0,000 & 37 \\
\hline 4,536 & 0,135 & 0,094 & 0,082 & 0,000 & 32 \\
\hline 4,536 & 0,135 & 0,094 & 0,082 & 0,000 & 35 \\
\hline 7,170 & 0,247 & 0,102 & 0,099 & 0,072 & 36 \\
\hline 7,170 & 0,247 & 0,102 & 0,099 & 0,072 & 50 \\
\hline 7,170 & 0,247 & 0,102 & 0,099 & 0,072 & 42 \\
\hline 10,110 & 0,232 & 0,119 & 0,075 & 0,648 & 84 \\
\hline 10,110 & 0,232 & 0,119 & 0,075 & 0,648 & 94 \\
\hline 10,110 & 0,232 & 0,119 & 0,075 & 0,648 & 87 \\
\hline
\end{tabular}

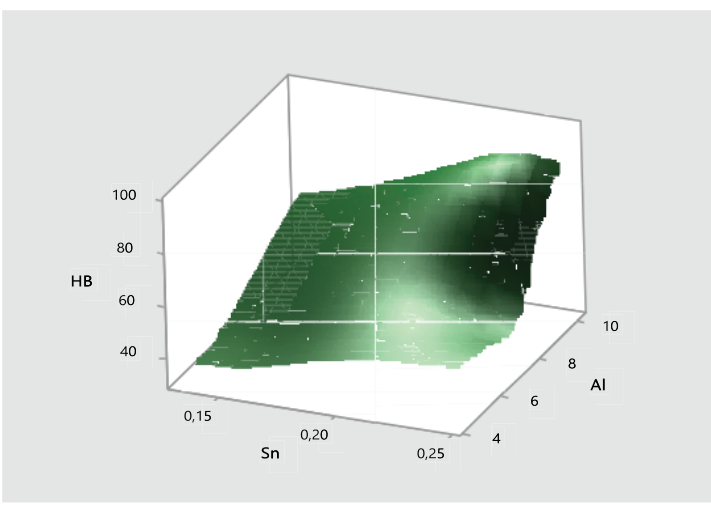

Figure 6

Grafica de superficie HB vs Al-Sn.

Se procede a encontrar la regresión de superficie de respuesta, inicialmente ajustando a un modelo totalmente cuadrado. A continuación, se muestran las tablas de 
varianzas y coeficientes codificados para un análisis RSM. Para describir la Tabla 11, es necesario saber el comportamiento y la incidencia que tiene cada elemento a la dureza

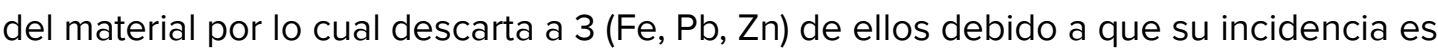
nula, si el valor $p$-value $<0,05$ indica que se puede desechar la hipótesis nula de que el coeficiente es igual a cero (no tiene incidencia) [25]. Por lo tanto, a la tabla anterior se observa un $p$-value $<0,05$, lo que afirma que los valores de Al y Sn tienen una alta incidencia en el comportamiento de HB.

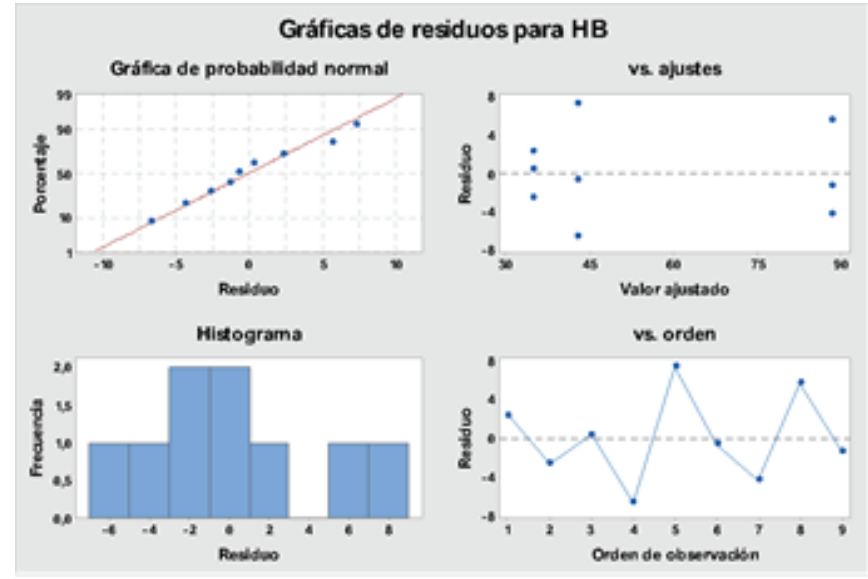

\section{Figure 7}

Residuos de la regresión software Minitab ${ }^{\circledR}$.

La Figura 7, muestra una distribución de residuos normal, lo que indica de igual forma que la regresión de superficie de respuesta es buena.

\section{Table 11}

Análisis de varianza.

\begin{tabular}{lllllll} 
Fuente & GL & SC Ajust. & MC Ajust. & Valor F & Valor $\mathbf{p}$ \\
\hline Modelo & 2 & 5029,6 & 2514,78 & 92,00 & 0,000 \\
\hline Lineal & 2 & 5029,6 & 2514,78 & 92,00 & 0,000 \\
\hline Al & 1 & 3706,6 & 3706,61 & 135,61 & 0,000 \\
\hline Sn & 1 & 602,2 & 602,15 & 22,03 & 0,003 \\
Error & 6 & 164,0 & 27,33 & & \\
\hline Total & 8 & 5193,6 & & &
\end{tabular}

Table 12

Coeficientes codificados.

\begin{tabular}{l|l|l|l|l|l} 
Término & Coef & EE del coef. & Valor T & Valor $\mathbf{p}$ & FIV \\
\hline Constante & 59,53 & 1,92 & 30,96 & 0,000 & \\
\hline Al & 39,56 & 3,40 & 11,65 & 0,000 & 2,54 \\
Sn & $-14,69$ & 3,13 & $-4,69$ & 0,003 & 2,54
\end{tabular}




\section{Table 13}

Resumen del modelo.

\begin{tabular}{l|l|l|l}
$\mathbf{S}$ & R-cuad. & R-cuad. (ajustado) & R-cuad. (pred) \\
\hline 5,22813 & $96,84 \%$ & $95,79 \%$ & $92,90 \%$
\end{tabular}

De la Tabla 13, se tiene que la correlación múltiple al cuadrado R2 $=95,79$ que es confiable y los dos compuestos son significativos de acuerdo a las pruebas $t$-value y $p$-value mencionadas anteriormente. El resultado de la regresión de superficie de respuesta manifiesta el comportamiento de los dos elementos en especial el aluminio de acuerdo al diagrama Pareto (Figura 8), cómo se comporta la dureza al incremento de la concentración.

$$
H B=5,70+14,19 x,-262,4 x,
$$

donde: $\mathrm{X} 1, \mathrm{X} 2$ son las concentraciones de $\mathrm{Al}, \mathrm{Sn}$ respectivamente, hay que acotar que en el modelo el estaño posee menos incidencia que el aluminio.

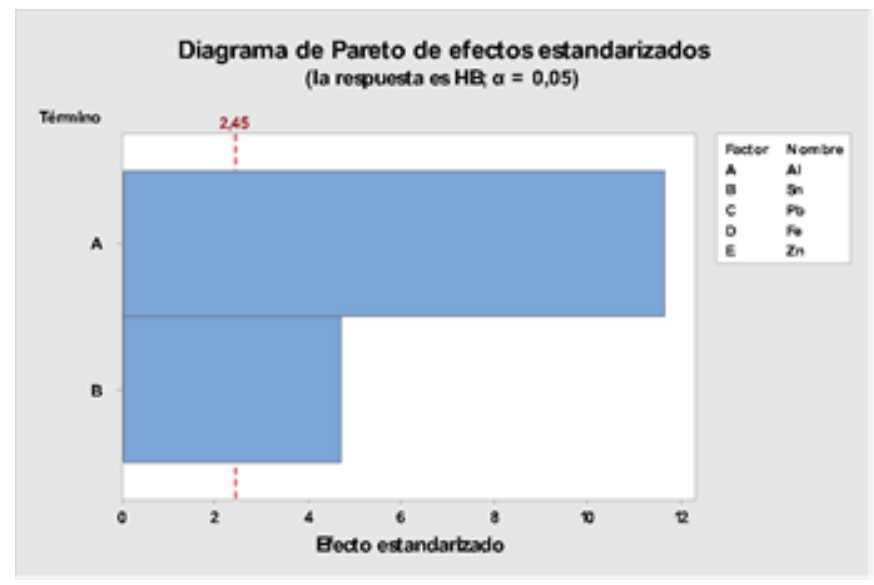

\section{Figure 8}

Diagrama de Pareto de efectos estandarizados.

En la Figura 9, se grafico la superficie de la funcion [12], decribiendo plano tridimensional afirmando que la influencia de la concentracion del elemento con mas incidencia en la ecuacion con un $p$-value de 0 , en este caso el aluminio, posee un comportanmiento $(\mathrm{K}=5,70)$ proporcional.

La grafica de contorno (Figura 10), muestra que a medida que el Al aumenta sube la dureza alcanzando un limite superior a los 10HBW, siempre y cuando el estaño no sea mayor al 0,16 wt.\% Sn. 


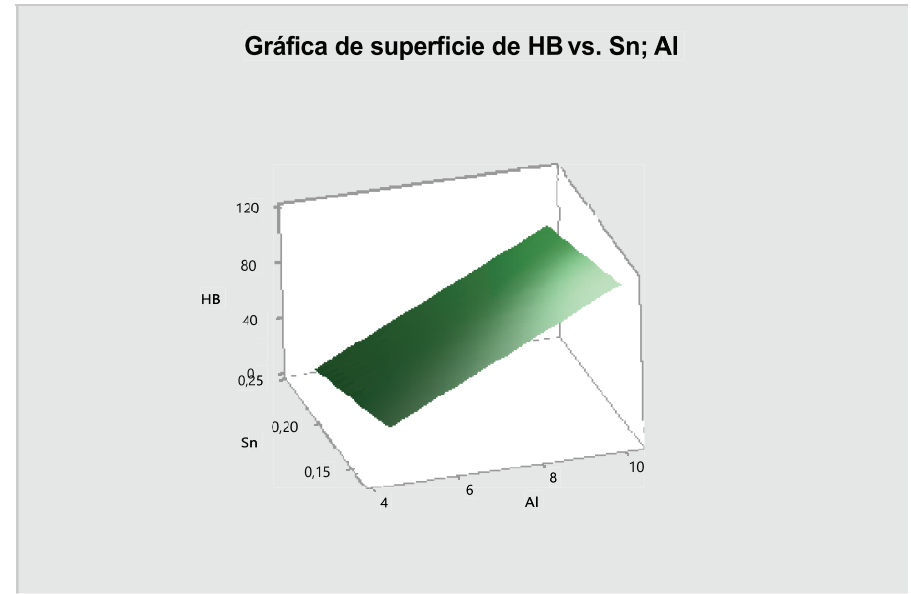

\section{Figure 9}

Grafica de superficie de la ecuación [12].

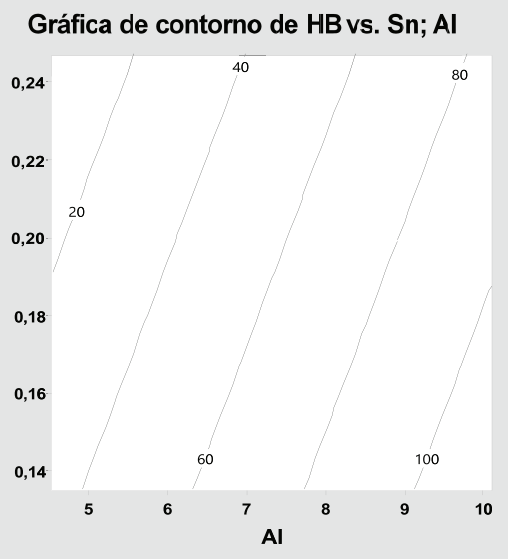

Figure 10

Contorno de superficie 2D (Al-Sn).

\subsection{Microscopia óptica y SEM}

En la microscopia electrónica de barrido SEM y para la microscopia óptica convencional se utilizó un microscopio electrónico Jeol JSM-IT100 y un Nikon eclipse E200, respectivamente.

La Figura 11a, se es una aleación con un contenido de 4,5 wt.\% Al, como bien se sabe las aleaciones con un contenido wt.\% $\mathrm{Al}<8$, presentan una única fase denominada $\alpha$ primaria, también se evidencia una inclusión de oxido o de la piel, costra que se expande en forma de piel oxidada y una inclusión de punto duro se originan por fundición en molde permanente. La Figura 11b, pertenece a una concentración de 7 wt.\% Al, de la misma forma se tiene una única fase $\alpha$, pero se evidencia la presencia de fases kappa, la presencia de estas fases podría darse a la presencia de Fe, dando origen 


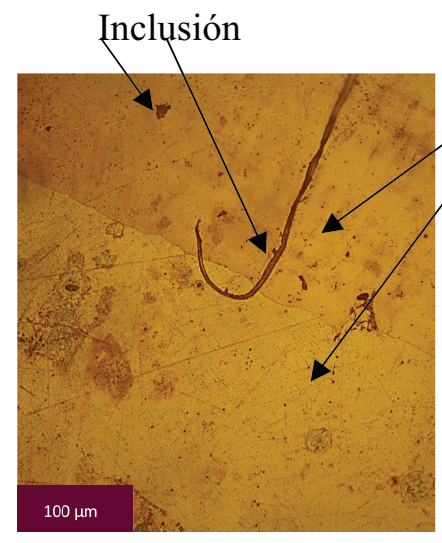

a.

\section{Figure 11}

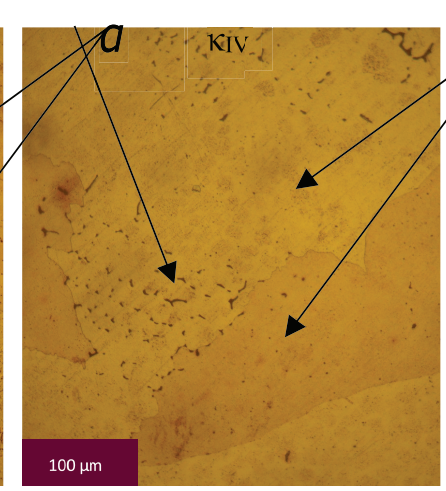

b.

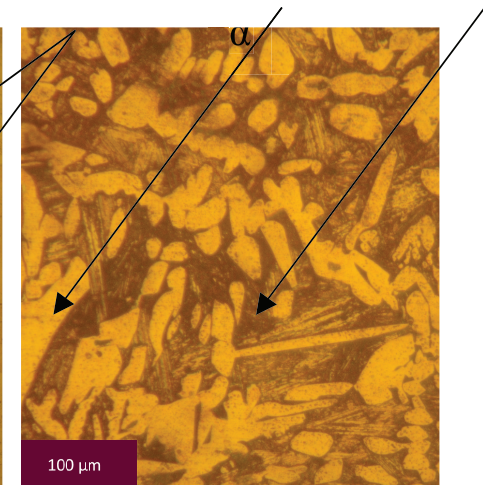

C.

Microscopia óptica a 200x (a) Cu-4,5 wt.\% Al, (b) Cu-7 wt.\% Al, (c) Cu-10 wt.\% Al.

a precipitados ricos en hierro en la fase alfa denominados fase $\mathrm{KIV}$, la solución solida $\alpha$ primaria, presenta una estructura cristalina FCC, es plástica, dúctil y rica en cobre [26]. En la Figura 11c, tenemos una microestructura multifásica que consiste en una solución solida $\alpha$ y una fase martensítica $\beta$, la fase $\beta$ o $\beta$, es esencialmente martensita.

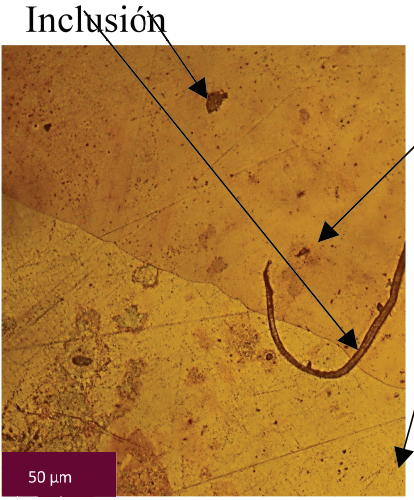

a.

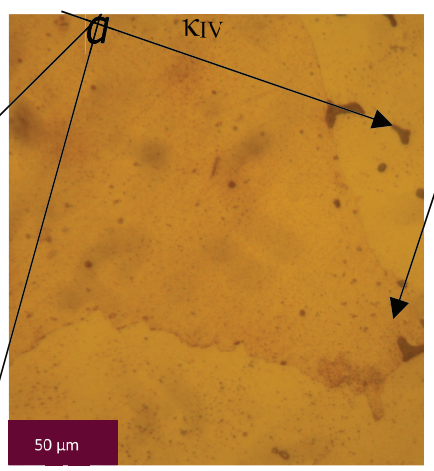

b.

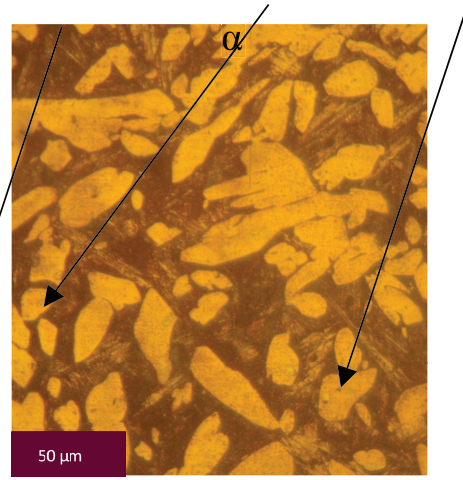

c.

\section{Figure 12}

Microscopia óptica a 500× (a) Cu-4,5 wt.\% Al, (b) Cu-7 wt.\% Al y (c) Cu-10 wt.\% Al.

La Figura 12, evidencia de forma más clara la presencia de las fases kappa, en forma de nódulos de menor tamaño en la Figura 12c, además se evidencia que la Figura 12a presenta dalos por el tiempo de exposición al grabado.

En la Figura 13 se puede presenciar la presencia de segregaciones de cobre en la fase primaria, también se puede apreciar la presencia de partículas globulares o precipitados kappa (KIV).

Algunas inclusiones lobulares, se presentan en la Figura 14a y también se evidencia porosidades por presencia de hidrogeno según [13], esto se debe a que hidrógeno 


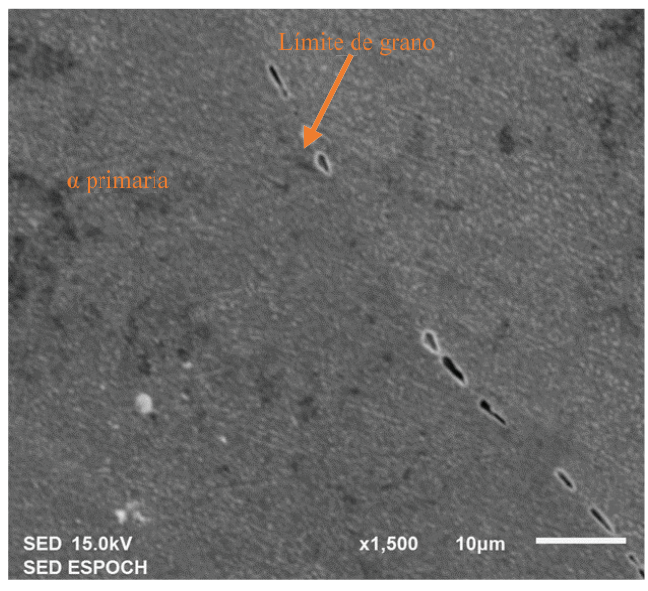

a.

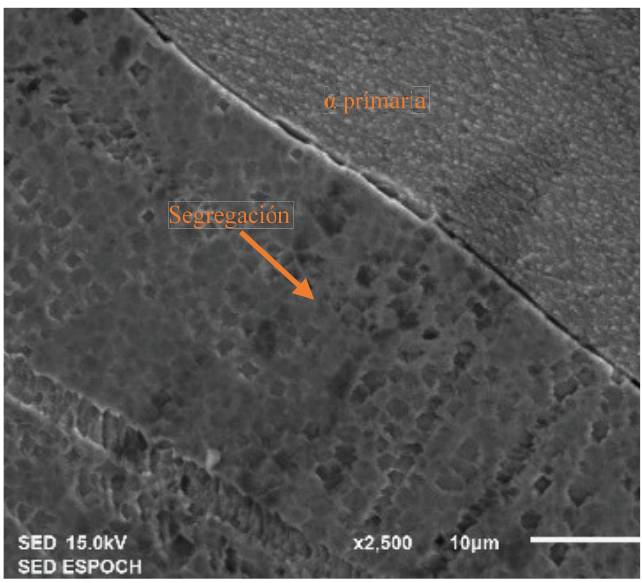

b.

Figure 13

Microscopia SEM (a) Cu-4,5 wt.\% Al 1,5 Kx; (b) Cu-4,5 wt.\% Al 2,5 Kx.

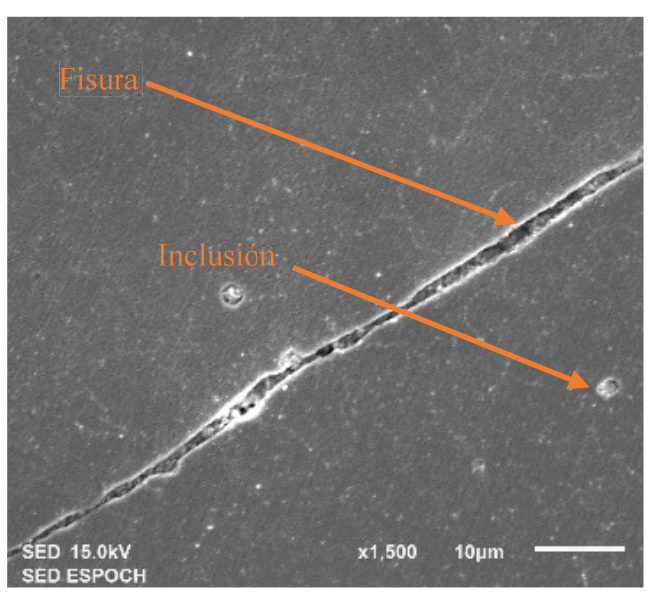

a.

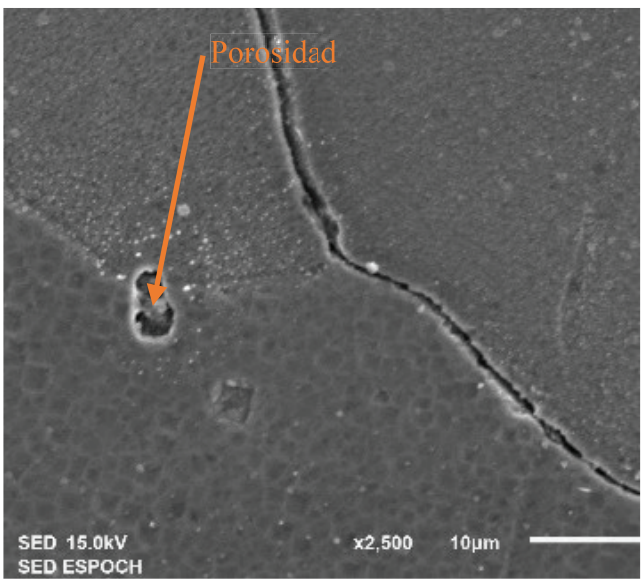

b.

\section{Figure 14}

Microscopia SEM (a) Cu-7 wt.\% Al 1,5 Kx; (b) Cu-7 wt.\% Al 2,5 Kx.

hace una contribución comparativamente pequeña a la nucleación de la burbuja, ya que se concentra relativamente poco por delante del frente de congelación que avanza, en comparación con los efectos combinados de oxígeno y carbono para formar CO en hierro y acero líquidos. La situación es muy paralela en las aleaciones de cobre líquido, donde el oxígeno controla la nucleación de los poros debido al mecanismo de quitanieves, mientras que el hidrógeno contribuye de manera desproporcionada al crecimiento debido a su mayor velocidad de difusión, claramente, si el oxígeno fuera alto, entonces los poros podrían nuclearse, pero no necesariamente crecerían a menos que hubiera suficiente hidrógeno presente. Por el contrario, si el hidrógeno fuera alto, los poros podrían no formarse si no hubiera oxígeno para facilitar la nucleación. Por lo 
tanto, el hidrógeno simplemente permanecería en solución en la colada. Los mismos argumentos se aplican, por supuesto, a las funciones del hidrógeno y el oxígeno en las aleaciones a base de cobre. Por otro lado, la presencia de una fisura se debe a la presencia de turbulencia o a burbujas de gran tamaño a momento de colar o por un impacto exterior lo que generan fracturas en la superficie [27], como se muestra en la Figura 14.

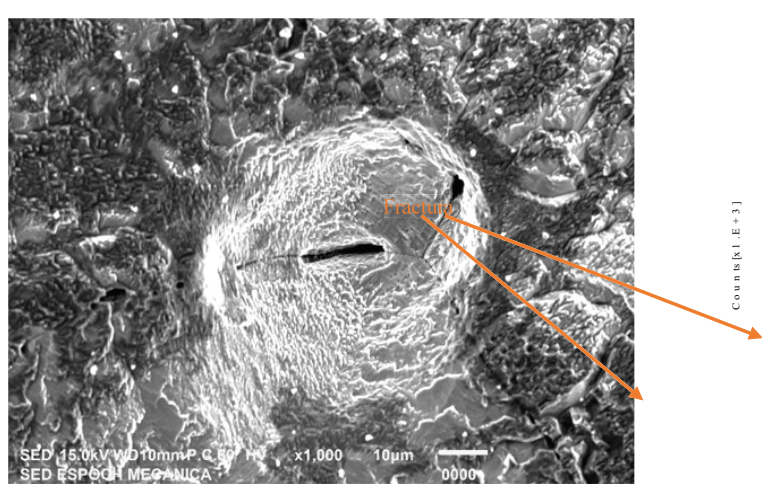

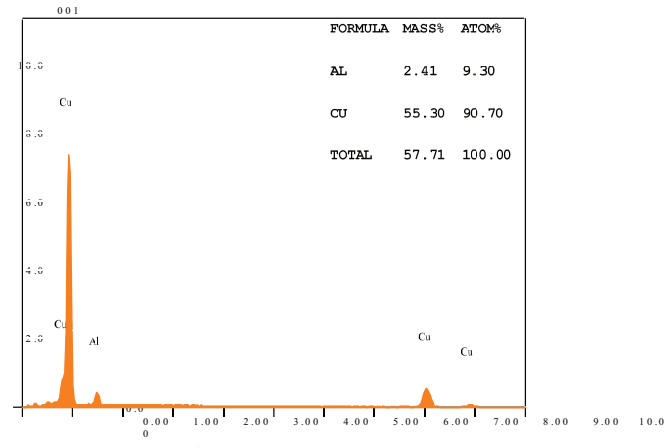

b.

\section{Figure 15}

(a) Morfología SEM de una fractura producida por la propagación de la grieta debido a un ataque corrosivo electroquímico. (b) EDS de la grieta (Cu-7 wt.\% Al 1,0 Kx).

Las fisuras que se originan en el proceso de manufactura de las aleaciones Cu-Al, pueden dar origen a una fractura debido a la propagación de los esfuerzos además que éstas son expuestas a medios corrosivos contribuyen notablemente a la propagación de una corrosión intergranular y Pitting paralelamente.
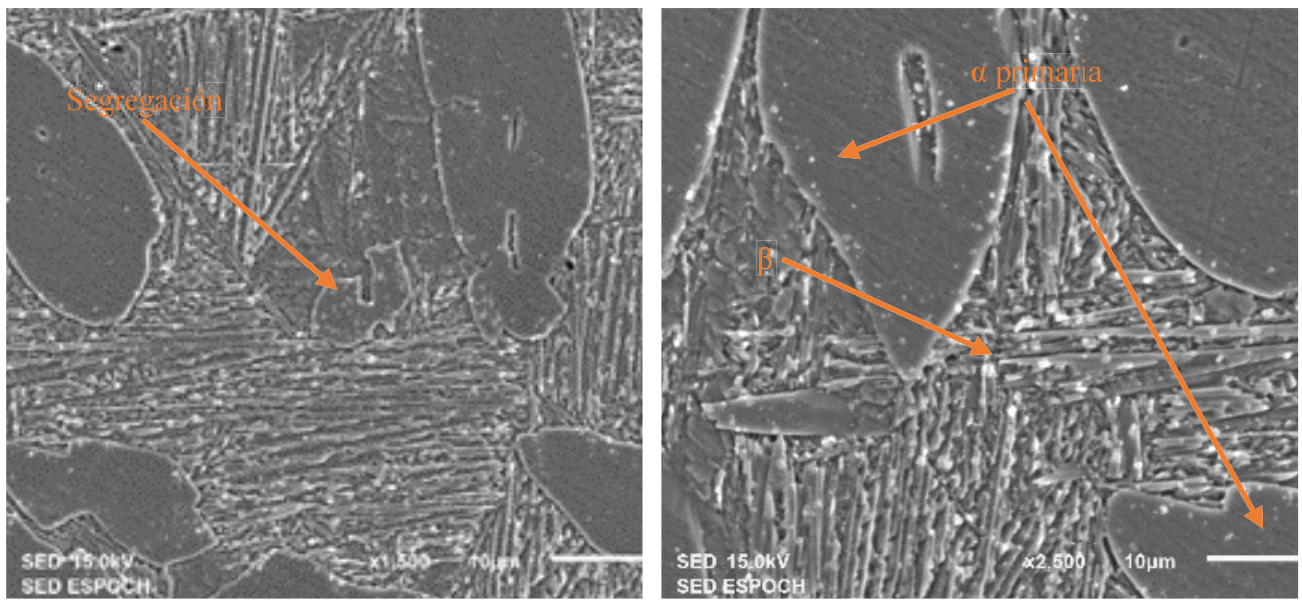

a.

b.

Figure 16

Microscopia SEM (a) Cu-10 wt.\% Al 1,5 Kx; (b) Cu-10 wt.\% Al 2,5 Kx. 
La microscopia SEM de la aleación Cu-10 wt.\% Al, muestra claramente como para un aumento de la concentración de aluminio en un 10\% wt. Ha creado una matriz similar a la de los aceros en forma martensítica aportándole mayor dureza como se evidencia en la Tabla 8.

En general los bronces con un contenido mayor al $8 \%$ de aluminio la fase $\beta$ se transforma en una microestructura tipo martensita, cuya propiedad es aumentar la resistencia a tensión y dureza a indentación, en las micrografías se encuentran dos tipos de fases, la alfa $(\alpha)$ y la $(\beta)$ demostrando lo observado por Morral y Jimeo [28].

\section{Conclusiones}

Las principales dificultades encontradas en la manufactura de este tipo de aleaciones de cobre se encuentra en la diferencia de puntos de fusión que originan la pérdida de masa de aluminio, haciendo difícil conseguir las composiciones deseadas, además se debe manifestar que la presencia de gas sobre el metal líquido; como el hidrogeno, que es soluble con el cobre y crece rápidamente a medida que aumenta la temperatura, mezclándose durante la solidificación y formando porosidades que afectan al rendimiento de la aleación en sus propiedades mecánicas.

La fundición de las aleaciones de Cu-Al al igual que las aleaciones de acero se deben realizar teniendo su aplicación ya que dependiendo de la forma del grano la aleación obtendrá varias propiedades, en el caso de que tengan una distribución equiaxial sería adecuados para aplicaciones como: motores de vehículos, y si son columnares podrían utilizarse para alabes de turbinas.

El tiempo de llenado del molde tiene influencia en la obtención de las muestras, si se cola muy rápido o muy lento se obtiene piezas defectuosas; si es lento produce solidificación prematura y si es rápido aumenta la presencia de grietas por gas o turbulencias.

Las aleaciones Cu-Al que se fabricaron en el presente trabajo evidencian metalográficamente una estabilidad microestructural para concentraciones en peso del $4 \%$ y $7 \%$ de aluminio presentándose como aleaciones (Cu-Al) de estructura monofásica alfa ( $\alpha$ ), con algunos precipitados Kappa (K), por otro lado, la aleación con mayor concentración de aluminio tiende a generar una microestructura martensítica beta $(\beta)$.

Metalográficamente se evidencia que la concentración de aluminio genera cambios en la microestructura en la cual, a mayor concentración de este elemento se tiende a generar una microestructura tipo martensítica. El análisis de composición química muestra la presencia de Sn, lo que podría generar una aplicabilidad en la fabricación de elementos sensibles de los manómetros, conectores eléctricos y resortes de alta precisión, pues el estaño al ser un componente importante en el manejo de la soldadura, 
se adhiere con mayor facilidad y a temperaturas considerablemente inferiores a sus puntos de fusión, generando mayores ventajas.

Se concluye que el cambio microestructural comienza una vez que se ha pasado el límite de solubilidad de la solución solida $\alpha$, a partir de este punto el comportamiento microestructural cambiará de monofásico a multifásico con fases como $(\alpha+\beta)$ y la descomposición de la fase beta en el eutectoide en $(\alpha+\gamma 2)$, este cambio microestructural aporta buenas propiedades de dureza, pero a medida que la matriz se endurece tiende a ser más frágil.

Las propiedades de dureza en los bronces se ven ampliamente influenciadas por el contenido de aluminio en la aleación, obteniéndo un aumento en el valor de la dureza a medida que aumenta la concentración de aluminio, es decir presentan un crecimiento lineal en su comportamiento ante la indentación, generando valores de dureza del orden de 35 HBW5/62,5 para (Cu-4,5 wt.\% Al); 43 HBW5/62,5 para (Cu-7 wt.\% Al); 88 HBW5/62,5 para (Cu-10 wt.\% Al). Estas fundiciones al ser únicamente (Cu- Al), no presentan durezas altas, debido a que su tamaño de grano, en especial las alecciones con 4,5 -7,1\% son muy grandes, y para subir su resistencia a la indentación requiere de un elemento aleante extra que refine su grano como es $\mathrm{Fe}$ y $\mathrm{Ni}$, estas aleaciones tienden a generar una fase precipitante kappa entre los $\mathrm{Ni}, \mathrm{Fe}, \mathrm{Al}$ ).

\section{References}

[1] Ruiz, AR. Estudio de la corrosíon electroquímica en los bronces de aluminio con níquel [Master’s Thesis]. Madrid: Univerisad de Cantabria; 2014.

[2] Herenguel, J. Metalurgia Especial. Urmo; 1971.

[3] Avner, SH. Introducción a la metalurgia física. Mexico: Mc Graw Hill; 1977.

[4] Vega C, et al. Mejoramiento de la aleación de bronce al aluminio, revista latinoamericana de metalurgia y materiales. 2009. Disponible en: http://www.rlmm. org/archives.php?f=/archivos/S01/N3/RLMMArt-09S01N3- p1311.pdf.

[5] King F. El aluminio y sus aleaciones. Mexico: Limusa, 1992.

[6] Slater JC. Atomic radii in crystals. The Journal of Chemical Physics. 1964;41(10): 31993204.

[7] Tuthill AH. Materials performance focus on seawater corrosion. NACE International; 1987. Disponible en: https://www.copper.org/publications/pub_list/pdf/ A7032-seawater- corrosion.pdf.

[8] Klement JF, Maersch RE. Use of alloy additions to prevent intergranular stress corrosion cracking in aluminum bronze. Corrosion. 1960;16(10):519-522

[9] Turhan H, Aksoy M, Kuzucu V, Yıldırım MM. The effect of manganese on the microstructure and mechanical properties of leaded-tin bronze. Journal of Materials Processing Technology. 2001;114(3):207-211. 
[10] Cengel YA, Boles MA. Termodinámica. Séptima. DF: Mcgraw- Hill/Interamericana Editores, S.A.; 2012.

[11] Askeland, DR. Ciencia e Ingenieria de los materiales. Mexico: Thomson, International Editors; 1998.

[12] Shackelford, JF. Introducción a la ciencia de materiales para ingenieros. Madrid: Pearson Educacion, S.A.; 1998.

[13] Campbell, J. Complete casting handbook. Oxford: Elsevier; 2011.

[14] Rodríguez J, Martínez L, Romero JCR. Procesos industriales para materiales metalicos. Segunda. Madrid: Vision Net; 2006.

[15] Bohler. Catalogo de materiales. Quito: Bohler; 2016.

[16] Cengel Y, Cimbala J. Mecánica de fluidos. D.F:McGraw-Hill;2006.

[17] Mott, R. Mecanica de fluidos. Mexico: Pearson; 2006.

[18] Jelínek P, Elbel T. 2010. Chvorinov's rule and determination of coefficient of heat accumulation of moulds with non-quartz base sands. Archives of foundry engineering. 2010:10(4):77-82.

[19] Ares A, Rodriguez C, Schvezov C, Rosenberger M. Structural characterization and dimensional analysis during directional solidification of Al- $\mathrm{Cu}$ Alloys. Procedia Materials Science. 2012;1: 72-79.

[20] Almeida MB, et al. Response surface methodology (RSM) as a tool for optimization in analytical chemistry. Talanta. 2008;76(5):965-977.

[21] Esparza C. Optimizacion de un sistema de alimentacion para llenado de moldes con aleacion de aluminio [Doctoral thesis]. Mexico;2003.

[22] Tao X. Effect of $\mathrm{Fe}$ and $\mathrm{Ni}$ contents on microstructure and wear resistance of aluminum bronze coatings on 316 stainless steel by laser cladding. Surface \& Coatings Technology. 2018;342:76-84.

[23] Chiavari C, Colledan A, Frignani A, Brunoro G. Corrosion evaluation of traditional and new bronzes for artistic castings. Materials Chemistry and Physics. 2006;95(23):252-259.

[24] Rosario F, Villacorta L, Falconi V, Rengifo S. Tratamiento térmico de los bronces aluminio complejos, transformación martensítica y la fase kappa - templabilidad. Revista del Instituto de Investigacion (RIIGEO). 2011;14(27):65-72.

[25] Montgomery DC, Runger GC. Applied statidtics and probability for engineers. Limusa: Wiley; 2018.

[26] Jahanafrooz A, Hasan E, Lorimer GW, Ridley N. Microstructural development in complex nickel-aluminum bronzes. Metallurgical Transactions. 1983,14:1951-1956.

[27] Emamy M, Campbell J, Abbasi R, Kaboli S. Fluidity of Al based metal matrix composites containing $\mathrm{Al} 2 \mathrm{O} 3$ and $\mathrm{SiC}$ particles. International Journal of Cast Metals Research. 2009,6(22):430-437. 
[28] Morral FR, Jimeo E, Molera P. Metalurgia general. Barcelona: Reverté; 1985. 